\title{
Une autre logique. Sur les Objets mathématiques de Man Ray
}

Another Logic. On the Mathematical Objetcs of Man Ray

\section{Éric Brunier}

\section{OpenEdition}

\section{Journals}

Édition électronique

URL : https://journals.openedition.org/imagesrevues/10995

DOI : 10.4000/imagesrevues.10995

ISSN : 1778-3801

Éditeur :

Centre d'Histoire et Théorie des Arts, Groupe d'Anthropologie Historique de l'Occident Médiéval, Laboratoire d'Anthropologie Sociale, UMR 8210 Anthropologie et Histoire des Mondes Antiques

\section{Référence électronique}

Éric Brunier, « Une autre logique. Sur les Objets mathématiques de Man Ray », Images Re-vues [En ligne], 19 | 2021, mis en ligne le 30 janvier 2022, consulté le 28 février 2022. URL : http:// journals.openedition.org/imagesrevues/10995; DOI : https://doi.org/10.4000/imagesrevues.10995

Ce document a été généré automatiquement le 28 février 2022.

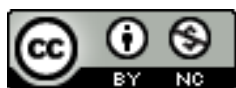

Images Re-vues est mise à disposition selon les termes de la Licence Creative Commons Attribution Pas d'Utilisation Commerciale 4.0 International. 


\section{Une autre logique. Sur les Objets mathématiques de Man Ray}

Another Logic. On the Mathematical Objetcs of Man Ray

Éric Brunier

À la mémoire d'H. Damisch avec qui il m'est arrivé d'extrapoler sur les Objets mathématiques. 

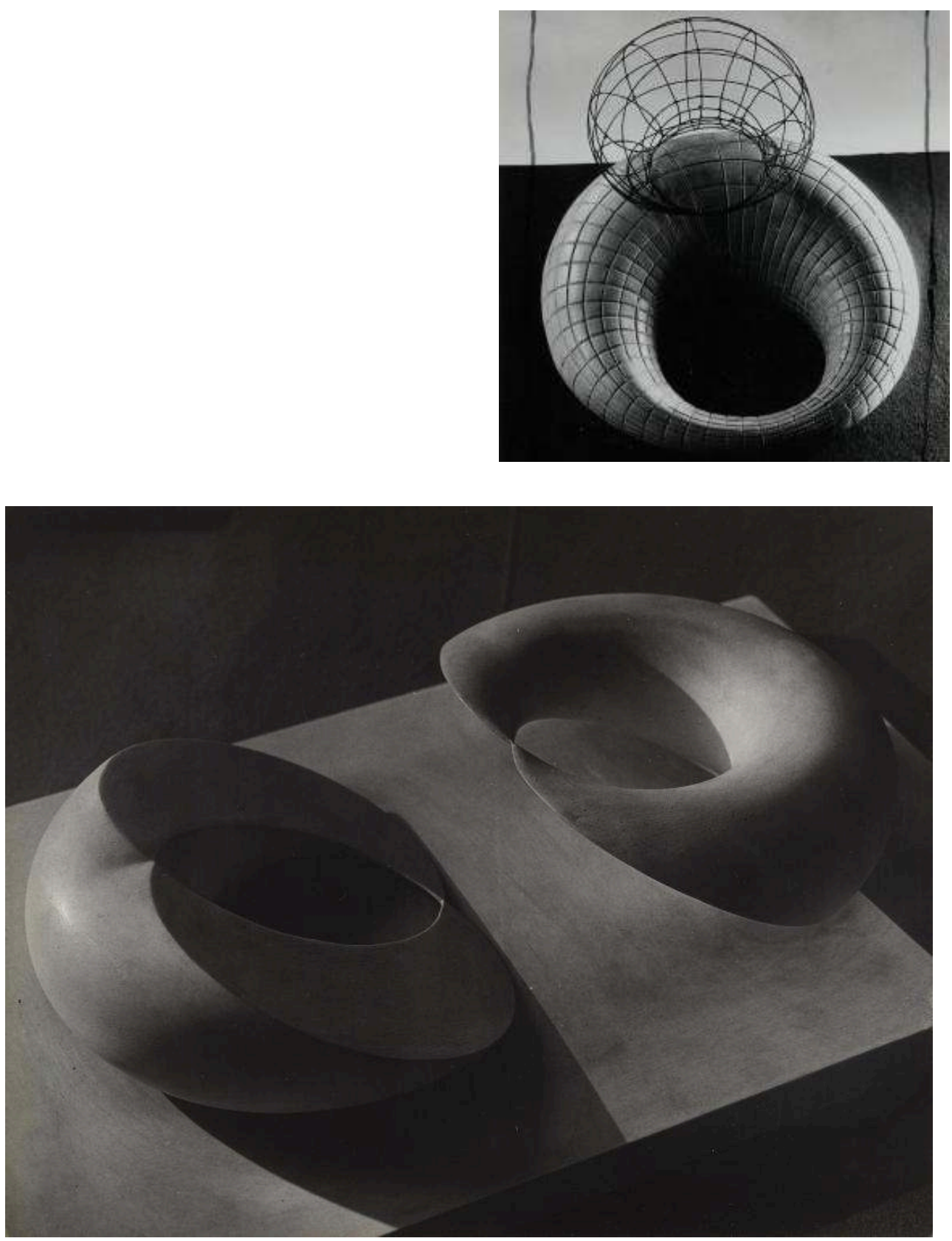

Man Ray, Objet mathématique (Plan bitangent à un tore le coupe suivant deux cercles. Théorème de Villarceau), 1934-36, épreuve gélatino-argentique, $30 \times 24$ cm, Paris, MNAM-Centre Pompidou. https://www.centrepompidou.fr/fr/ressources/oeuvre/cn7kdgE (c) Man Ray Trust / Adagp, Paris 
Figure 2

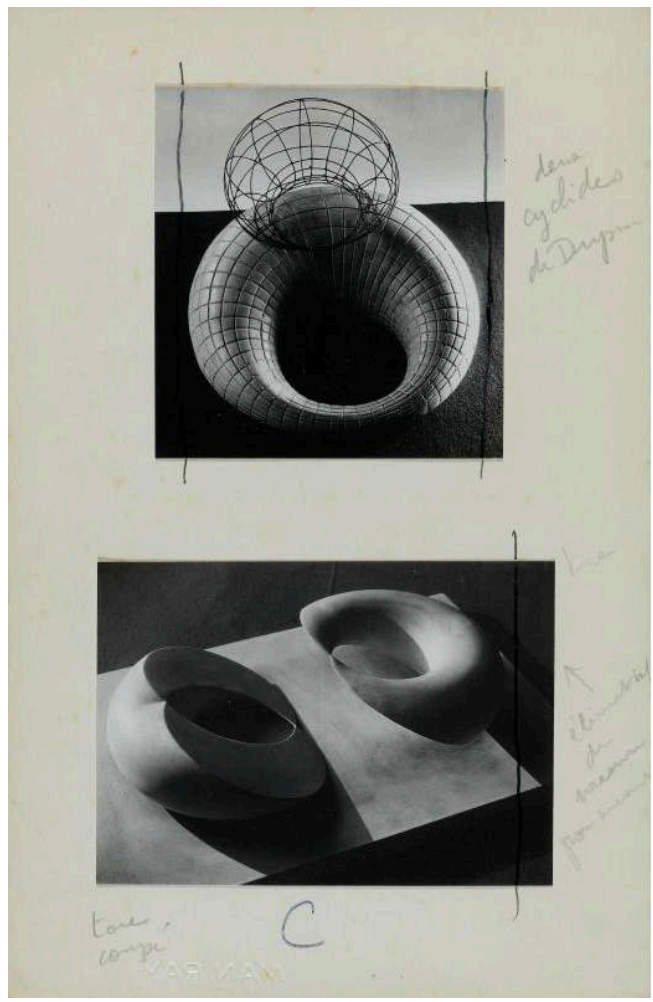

Man Ray, planche de l'album contenant trente-et-une photos avec des traits de recadrage de Man Ray et des annotations mathématiques ultérieures par Michel Collinet, 21,6 x 14,1 cm, Paris, MNAMCentre Pompidou.

https://www.centrepompidou.fr/fr/ressources/oeuvre/c4yn6L

(c) Man Ray Trust / Adagp, Paris 
Figure 3

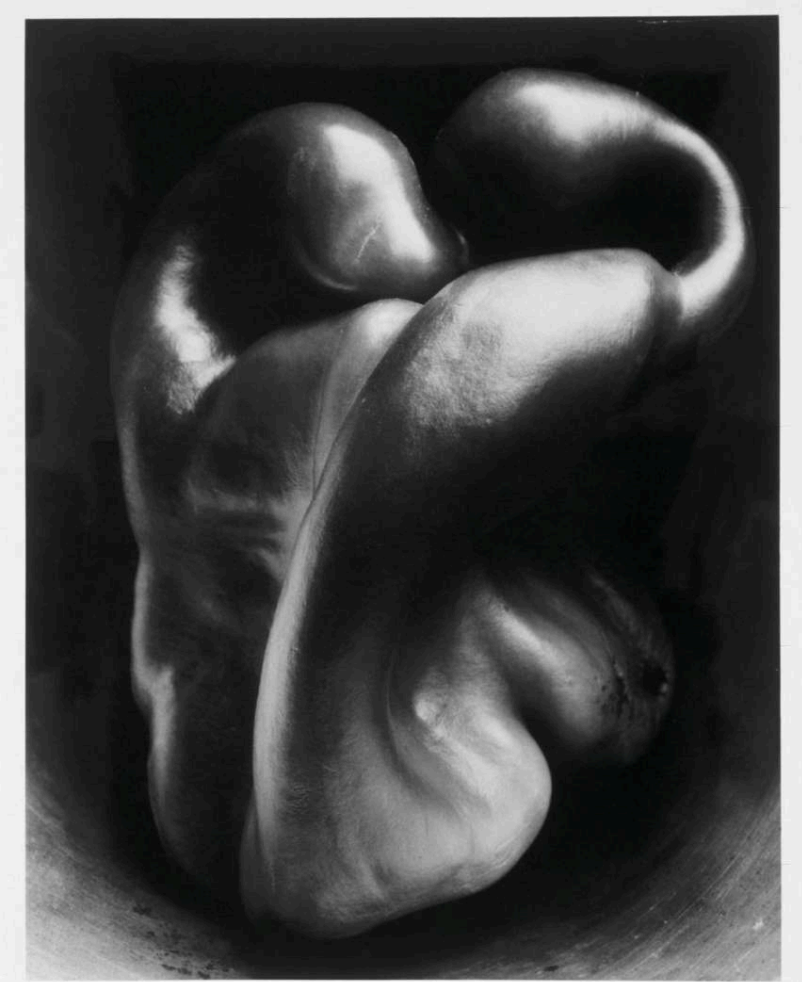

Edward Weston, Poivron n³0, 1930, épreuve gélatino-argentique, 24 x 19,2 cm, Cologne, Musée Ludwig.

https://museum-ludwig.kulturelles-erbe-koeln.de/documents/obj/05112921/rba_c012065

(c) Rheinisches Bildarchiv Köln, Zimmermann, Rolf, rba_c012065 
Figure 4

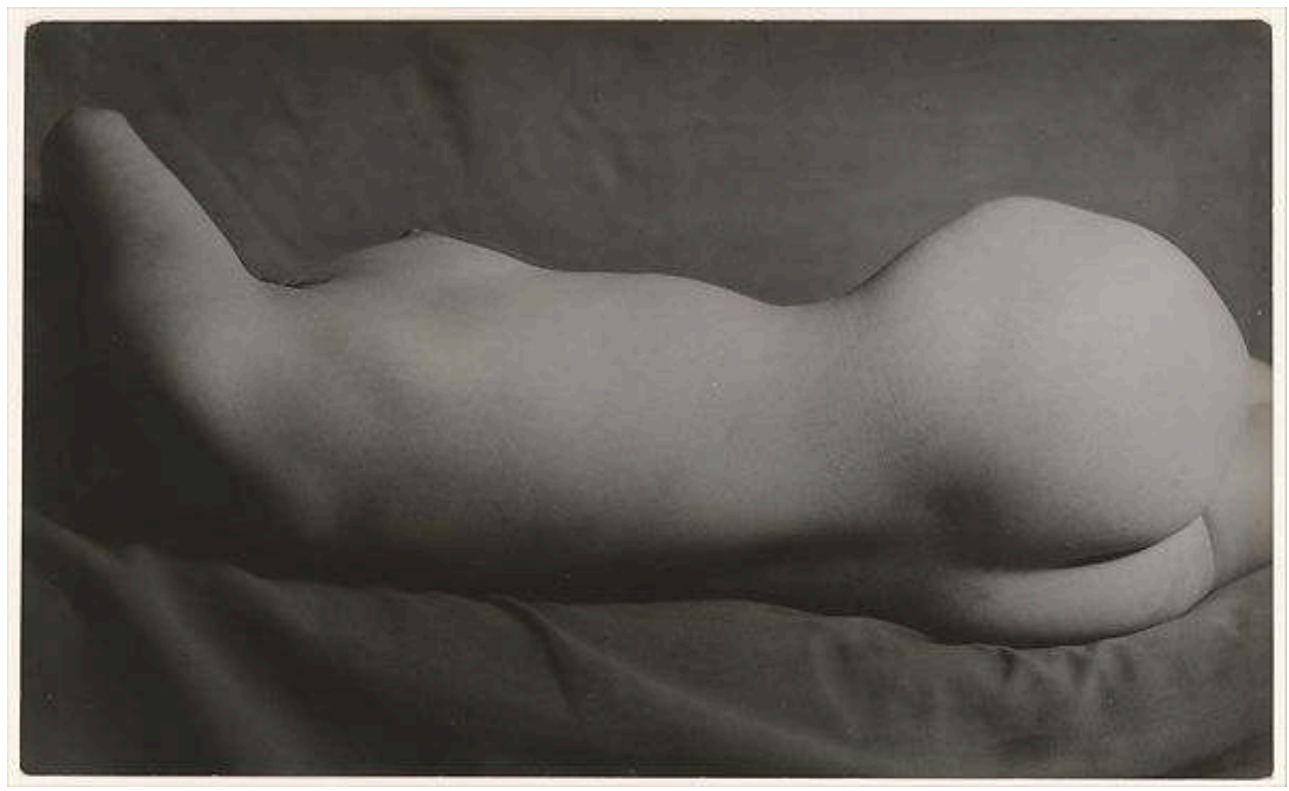

Brassaï, Sans titre, 1933, épreuve gélatino-argentique, 14,1 × 23,5cm, New York, Metropolitan Museum.

https://www.metmuseum.org/art/collection/search/288031?

searchField=All\&amp;sortBy=Relevance\&amp;who=Brassaï\%24Brassaï\&amp;ft=*\&amp;offset=0\&amp;rpp=20\&amp;pos=14 (c) Estate Brassaii Succession - Paris

1 Les photographies d'objets mathématiques que réalisa Man Ray à l'Institut Henri Poincaré de Paris vers 1934-35 relèvent du mystère. On ne connaît pas exactement la date des prises de vue, ni le déroulement de celles-ci. Il apparaît seulement qu'elles ont certainement été faites à plusieurs reprises et une fois que Man Ray eut reconstitué les conditions d'une prise de vue en studio : l'éclairage y est précis, fortement graphique et il souligne la surface tactile des objets, comme on peut le voir sur la figure 1. Le recadrage, comme le montre la différence entre le cliché visible sur un tirage contact (fig. 2) et l'épreuve pour Les Cahiers d'art, serrée sur les seuls objets, intensifie leur dramatisation en les décontextualisant, notamment par l'effacement partiel du fond devenu totalement anonyme, et par la dynamique du rapport au cadre qui leur donne force de figures : les objets tantôt acquièrent un caractère anthropomorphique, tantôt semblent une concrétion naturelle et monolithique. Ces photographies montreraient la fermeture des formes sur elles-mêmes. Le silence sur la genèse et la fonction des objets mathématiques gagne les photographies comme si le secret de la formation des modèles mathématiques touchait l'image entière. Les photographies de Man Ray regardent la pensée la plus abstraite comme force d'élaboration des formes. À la différence de la photographie d'un poivron comme la faisait Weston en 1930 (fig. 3), ou celle d'un corps humain partiel de Brassaï (fig. 4), la forme des objets mathématiques ne semble relever d'aucune intention explicite. Il n'y a pas ici d'écart entre la forme et le sujet, pas de motif, pas de reconnaissance puisqu'on ne reconnait rien. Alors, c'est que l'intention photographique elle-même est là, enfermée. Intention? On devrait plutôt dire formalisation, tant ces photographies se donnent comme formelles. On les considérera donc ici en tant que symptôme de la mise en forme visuelle et singulièrement photographique. On voudra y voir une pensée de la photographie sur elle-même, sur ses moyens de mise en forme. 
2 Pourtant ce n'est pas selon cette perspective que les photographies de Man Ray nous sont connues. Douze des trente-et-un clichés ont été tirés une première fois en 1936 pour le numéro de la revue Les Cahiers d'art ${ }^{1}$. Ces photographies illustraient d'une part l'article « Mathématiques et art abstrait » de Zervos, le directeur de la revue, d'autre part celui de Breton intitulé «Crise de l'objet ». La présence des photographies de Man Ray s'y explique en partie par la volonté de documenter une pratique de l'abstraction dans le domaine des mathématiques, pour l'article de Zervos. Quant à l'article de Breton, son attention se portait sur le statut des objets. Le numéro de la revue des Cahiers d'art intitulé «L'objet surréaliste » se faisait lui-même l'écho de l'« Exposition surréaliste d'objets » à la galerie Charles Ratton de Paris. Rappelons que l'enjeu à cette époque est l'extension du domaine de l'art. Grâce aux travaux de Bachelard sur le "nouvel esprit scientifique », Breton proposait dans son texte une extension similaire entre l'art et les sciences. Il s'agirait dans les deux cas de passer du donné au possible. La pensée moderne selon Breton, commune aux sciences et aux arts, y est décrite " comme une volonté d'objectivation sans précédent ${ }^{2}{ }^{2}$. L'important pour Breton est d'exalter la création d'objets, création que l'on trouve dans la nature, dans les sciences et dans les arts. Toutefois la conquête du possible que propose Breton y est moins une extension des capacités réelles des mathématiques et des arts que la traduction, la visualisation des trésors cachés de l'imagination humaine. Breton, à la suite de Man Ray, avait donc vu qu'un nouveau domaine du visible se faisait jour dans les objets mathématiques. Selon lui, ces objets, comme ceux plus communs qui se trouvent détournés de leur usage premier, attestent d'un " passage à la réalité " ${ }^{3}$, révèlent des tendances inconscientes. Leur puissance est de provocation.

Après ce préambule qui nous renseigne sur le contexte d'apparition et de réception de la série des Objets mathématiques, il s'agira d'en proposer une autre interprétation. On ne les considérera pas comme des illustrations, mais comme des œuvres de plein droit. Trois considérations nous amènent à cette proposition. Les deux premières sont d'ordre factuel, la troisième est théorique. On redira tout d'abord que les photographies de Man Ray sont étranges et que leur étrangeté n'a cessé de susciter l'intérêt et les interrogations. Man Ray lui-même s'en inspira dans les années 40 pour créer une série de peinture nommée Équations shakespeariennes. Un second élément à considérer est le rôle que jouent ces œuvres dans la transformation patrimoniale des modèles mathématiques. L'Institut Henri Poincaré vient de terminer une restauration de ses collections, le musée du Temps de Besançon a consacré une exposition à la collection du Laboratoire de Mathématiques de l'Université de Besançon ${ }^{4}$. On pourrait même avancer l'hypothèse que le glissement de dénomination de "modèle » vers " objet ", qui témoigne en faveur de la transformation patrimoniale, est un effet des photographies de Man Ray. Enfin, la troisième considération tient aux enjeux artistiques de cette série de photographies. Notre idée est la suivante: de la même manière que les modèles mathématiques jouent un rôle dans la pensée mathématique, les photographies de Man Ray se saisissent d'une partie de la pensée de la photographie sur elle-même. Pour autant, les deux places occupées ne sont pas les mêmes. Le modèle mathématique, comme on le verra, occupe un pôle dans un dispositif général nommé système formel. On l'exposera dans un premier temps. Les photographies de Man Ray sont quant à elles un symptôme, une singularité, c'est-à-dire qu'elles concentrent en un point unique deux tendances contraires. Il s'agit donc moins d'une dialectique que d'une topologie où se rencontrent d'un côté le domaine de la concrétion, de l'objet et du hasard comme le voulait Breton, et d'un autre côté le domaine du construit, de 
l'élaboré et de la recherche patiente comme le suggèrent les mathématiques. Ceci nous amènera à nous interroger sur ce que peut signifier formaliser dans le domaine de la photographie et des arts du visible.

4 Avant d'être des modèles photographiques, les objets photographiés par Man Ray sont des modèles mathématiques. Mais de quoi sont-ils les modèles ? Et surtout lorsque l'on parle de modèle en mathématiques, de quoi parle-t-on? À la première question, la réponse est à la fois simple et compliquée. Simple, car il s'agit de modèles d'équations, algébriques et différentielles, et que ces équations sont écrites sur les socles ou sur les corps géométriques. Compliquée car certaines étiquettes ont aujourd'hui disparu, que certains modèles se ressemblent fortement et que Man Ray a même parfois photographié non le modèle mais son support ${ }^{5}$. Ces modèles sont donc les réalisations visuelles d'équations chiffrées, en quelque sorte leur déploiement dans l'espace. Isabelle Fortuné a bien raison de citer Vuibert à propos de leur fonction didactique ${ }^{6}$. La traduction visuelle et en volume des équations, lorsque celles-ci sont devenues fortement abstraites et complexes (équations du $3^{\mathrm{e}}$ et du $4^{\mathrm{e}}$ degré par exemple) a été elle-même rendue possible par la géométrie descriptive mise au point par Monge au XVIII siècle. Ceci signifie que la fabrication du modèle obéit à des règles de dessin géométrique qui lui sont propres. Le principe didactique peut s'énoncer de la manière suivante: dans le même temps où l'étudiant acquiert les notions de géométrie lui permettant d'abord de réaliser l'épure de son solide (un dessin réglé par la géométrie descriptive), puis sa fabrication en volume, il met en œuvre les éléments de calcul algébrique impliqués par l'équation. Une autre fonction didactique pouvait être réalisée en visualisant une fonction, ce qui peut aider à en avoir l'intuition. Au niveau didactique, les modèles sont donc un élément de la dialectique entre la géométrie et l'algèbre. Depuis Descartes, la géométrie s'est algébrisée (on s'est mis non plus à manipuler les figures géométriques mais à les écrire sous forme d'équation comme par exemple celle du cercle: $x^{2}+y^{2}-R^{2}=0$, où $x$ et $y$ désignent deux variables et $R$ le rayon.). Ceci a permis d'élaborer et de comprendre des figures et des corps géométriques de plus en plus complexes. Puis à partir du milieu du XIX ${ }^{e}$ siècle, c'est l'algèbre qui s'est géométrisée pour gagner en compréhension des nombres. La fabrique de corps mathématiques étranges et complexes intervient au moment de la conquête de l'algèbre par la géométrie. C'est peut-être un facteur de ce nouveau développement des mathématiques modernes. Pour autant, un modèle mathématique ne remplit pas seulement une fonction didactique. Alain Badiou, dans son petit livre Le Concept de modèle ${ }^{7}$, propose de distinguer la notion de modèle de celle de concept. La notion est celle que l'on utilise quand on veut désigner l'original d'une copie ou lorsque l'on parle de modélisation. À certains égards, les objets photographiés par Man Ray, sont des modèles au sens de la notion; ils modélisent des fonctions, nous aident à en acquérir une représentation. Mais ce sont des modèles au sens fort du terme, dans le sens que les mathématiques auront donné à ce terme pour leur usage propre, ce que Badiou propose de distinguer sous le terme de concept. Celui-ci renvoie aux systèmes formels, concept aussi mathématique.

5 Il faut bien prendre garde au fait que si l'observation ou la construction de modèles mathématiques a des vertus pédagogiques, cela vient du fait qu'elles engagent à des opérations d'ordre mathématique et géométrique et non à un plaisir d'ordre esthétique. On ne demande pas aux étudiants de contempler les modèles, puis de les reconstruire, mais de les penser en mathématiciens. Ils figurent mathématiquement des opérations de l'algèbre que les étudiants s'efforcent d'acquérir. Les modèles servent 
donc de truchement dans l'acquisition d'un savoir mathématique. Redisons les choses simplement : les modèles donnent à voir, aux moyens des surfaces et des lignes, des équations qui sont des opérations sur des nombres. Ils en sont comme une traduction ou une illustration déterminée et précise. Le langage mathématique parle à ce sujet d'interprétation. Mais à l'inverse, on peut passer du donné empirique, par exemple une sphère, à son expression sous forme d'équation. Le langage mathématique parle alors de formalisation. L'ensemble forme un système formel. Au sein de celui-ci on distingue donc le domaine de la théorie qui est une syntaxe (ce sont des lettres et des opérations comme dans une équation) et le domaine du modèle qui est une sémantique (ce sont des objets). Passer du modèle à sa théorie s'appelle formaliser, et parcourir le chemin inverse, interpréter ${ }^{8}$. Le système formel peut se schématiser sous la forme suivante ${ }^{9}$ :

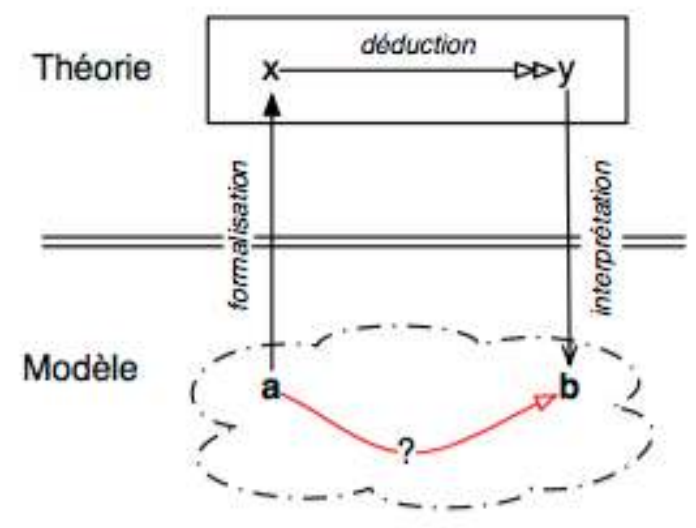

Dans ce système, la syntaxe est chargée de faire apparaître des relations qui demeurent cachées dans le modèle. Ces relations relèvent, pour les mathématiques, de la logique déductive. On peut ainsi, à partir d'un modèle que l'on a formalisé, effectuer des opérations syntaxiques et transformer le modèle initial. En fait la syntaxe a avant tout pour fonction de calculer la vérité des propositions. En cela son propos est uniquement logique. Par ailleurs, comme elle s'écrit essentiellement avec des lettres, elle a une très grande généralité. Dans ce sens on peut dire qu'elle est abstraite. Elle l'est aussi en un autre sens. Dans les formules, seules comptent les relations entre les symboles (les lettres et les opérateurs). La suspicion sur la réalité sensible des symboles a donc pu être écartée. Roger Martin écrit: «Le symbole est un être abstrait et [...] les signes tracés sur le papier ou sur le tableau servent seulement à l'évoquer ${ }^{10}$. " Néanmoins, dire que les symboles sont abstraits, cela ne veut pas dire qu'ils sont immatériels. Bien au contraire, la syntaxe formelle est une syntaxe matérielle. Alain Badiou, par exemple, compare le symbolisme arithmétique de la syntaxe au «tube à vide» ou à «l'accélérateur de particules» de la physique. Il écrit: «il s'agit d'une matérialité “rigide", manipulable et ouverte ${ }^{11}$. » Le point important est qu'au niveau de la syntaxe, les relations entre symboles sont mises au jour, que les opérations sont contrôlables comme dans un dispositif expérimental et que la seule réalité d'un symbole est sa valeur ou sa relation avec un autre symbole. Nous pouvons alors récapituler les caractéristiques du symbole formel : il est matériel, manifeste et discret. Le modèle, de son côté, sera alors caractérisé comme concret, latent et continu.

7 Avant d'étudier ce qu'impliquent les relations entre les deux domaines, nous allons préciser ce qu'est un modèle et à quelles conditions il l'est. Nous pouvons retenir l'idée qu'il faut distinguer le concept de la notion. Celui-ci, nous dit Alain Badiou, est mathématique. Qu'est-ce que cela signifie exactement? Quand on regarde un modèle 
mathématique, c'est-à-dire un objet, un corps géométrique, en quel sens peut-on dire que l'on regarde un concept mathématique ? La question invite à distinguer, à propos de ces modèles, leur usage didactique, ou aussi bien esthétique aujourd'hui, de leur réalité mathématique. Dans le premier cas, on a affaire à la notion de modèle. Leur réalité mathématique (ce en quoi ces modèles sont bien des concepts mathématiques) relève quant à elle de l'axiomatisation géométrique nécessaire à leur construction. C'est là un point fondamental que soulignent Roger Martin et Alain Badiou : la sémantique nécessite une axiomatique. En effet, pour interpréter une syntaxe, il faut que dans le modèle correspondent aux lettres et aux formules de la syntaxe des éléments d'une réalité spécifique. Dit autrement, les modèles que Man Ray photographie ne sont pas seulement des aspects, des formes abstraites, ce sont des figures géométriques. Dans l'autre sens, la formalisation s'exerce sur des objets ou des régions mathématiques déjà axiomatisés. Par exemple, Descartes va développer la formalisation de la géométrie qu'Euclide aura au préalable axiomatisée. Il est donc faux de penser que l'on peut donner une formule algébrique de n'importe quelle forme naturelle. On aboutit finalement à cette idée que le concept de modèle nous dit qu'un modèle peut être un modèle d'une formule syntaxique à condition d'être lui-même mathématique. De la même manière François Nicolas, soutient que dans le monde-Musique, le solfège formalise le corps-accord musical et non pas n'importe quel son ${ }^{12}$.

8 Au premier abord, cela paraît compliqué et il semble que les chemins empruntés sont bien longs pour ne pas avancer beaucoup. La formalisation mathématique s'occupe de modèles mathématiques, la musique de modèles musicaux et la photographie, par extension, de modèles photographiques. Dit ainsi, cela n'a pas grand sens. Entre la théorie et le modèle, la différence ne tient pas seulement à l'écriture discrète de l'une et au donné concret de l'autre. Outre le fait qu'on ne pratique pas les mêmes mathématiques au niveau de la théorie et de la sémantique, ou pas la même musique (il semble assez évident que la composition d'une symphonie se déroule au niveau de la théorie, quand son interprétation s'effectue au niveau du modèle), le système formel nous permet de penser que la logique de la syntaxe n'est pas la logique générale que l'on a à l'esprit, mais une logique particulière, adéquate à son modèle. Cela nous oblige donc à penser pour chaque système formel une syntaxe qui lui est propre. La relation entre la théorie et le modèle n'est donc pas une imitation, un décalque abstrait ou une simple traduction. Théorie et modèle interagissent l'un sur l'autre. Un second point est l'effet produit par la formalisation sur le modèle. Pour qu'il y ait formalisation, il faut que le donné soit transformé en modèle. Ainsi Badiou peut écrire: «le concept de modèle ne désigne pas un dehors à formaliser, mais un matériau mathématique à éprouver ${ }^{13}$.» Au sein du système formel se trouve une dialectique de l'unité, du même et de la différence. Eu égard aux photographies de Man Ray, on peut la penser comme l'unité du concret et du sensible que donne à voir la lumière qui enveloppe les objets, et la différence que crée l'armature d'un corps géométrique.

Cette conception rigoureuse que nous donnent les mathématiques du système formel, il nous faut maintenant l'étendre aux arts du visible et notamment à la photographie. Les photographies de la série réalisée par Man Ray nous semblent particulièrement indiquées pour une telle entreprise. On soutient en effet, que les photographies de modèles mathématiques ont pour sujet de montrer l'interrelation entre la forme et les choses, entre la syntaxe et la sémantique photographique. On rencontre toutefois trois difficultés à une telle extension. Sur quels éléments peut-on s'appuyer pour dire qu'il y a dans ces photographies un modèle photographique? Dans un système formel, en 
effet, le modèle appartient au même domaine que la théorie. La seconde difficulté tient quant à elle à la syntaxe. Quelle est celle de la photographie sachant qu'elle doit relever de la matérialité de la lettre ? Enfin la troisième difficulté tient à la généralisation que l'on peut attendre de l'étude de ce système formel. On pose en effet, comme condition de la solidité de notre propos, que l'étude du système formel tient dans l'adéquation entre la sémantique et la syntaxe. Comment passer d'une description particulière à une proposition plus générale? Si nous ne pouvions généraliser notre propos, nous aurions là affaire à un cas particulier et notre enquête eut été bien longue pour un résultat aussi maigre. Les réponses à toutes ces questions, que nous exposerons de manière isolée pour des raisons de clarté, se trouvent dans le fait que la relation entre la syntaxe et la sémantique est dialectique et dynamique. Elles s'ajustent l'une à l'autre de manière quasiment continue. Il faut comprendre que la syntaxe n'est pas un simple codage du réel mais qu'elle profite des développements du modèle. Et réciproquement, le modèle s'approfondit et se complexifie à mesure de sa syntaxe ${ }^{14}$.

10 Avant de répondre à nos trois interrogations, on donnera rapidement deux exemples de système formel d'un art. Le premier exemple est celui de la musique. François Nicolas en a donné une version théorique développée dans les deux premiers volumes de son Monde-Musique. Le sous-titre de chacun des volumes indique clairement ce qui relève de la sémantique et ce qui relève de la syntaxe. Le $1^{\text {er }}$ volume s'intitule " L'œuvre musicale et son écoute » et le $2^{\mathrm{e}}$, "Le monde-Musique et son solfège ». La formalisation proposée par François Nicolas repose bien sur la distinction entre les opérations d'écriture d'un côté et la structuration de l'œuvre de l'autre. On retrouve cette idée que la syntaxe est générale, le solfège utilisé d'une œuvre à l'autre étant le même, alors que la sémantique est propre à chaque œuvre. Un autre point important est que le modèle musical est structuré par l'écoute musicale. C'est l'écoute musicale, différente de l'écoute en général et de l'audition, qui structure l'œuvre, en quelque sorte l'axiomatise. Chaque œuvre particulière possède sa propre écoute musicale, laquelle est manifeste dans la partition, cette syntaxe de la musique ${ }^{15}$. Livre en main, on ne peut que chercher à éprouver la dimension structurante du « moment-faveur ». Si, grâce au solfège, l'existence d'une syntaxe musicale semble ne soulever aucun problème, la mise au jour d'une sémantique semble plus difficile à admettre. Quand on comprend que la sémantique, finalement, est la manière dont une œuvre musicale se déploie dans une écoute à l'œuvre, dont l'auditeur est le destinataire actif, que cette écoute est ellemême sujette à l'interprétation des musiciens, la chose devient plus facile à saisir.

11 Mais, pour les œuvres qui relèvent du visible, l'affaire semble encore plus compliquée. En effet, la musique partage avec les mathématiques le fait que leur terrain leur est propre: la syntaxe et la sémantique des mathématiques sont de part en part mathématiques, arithmétique et géométrie, par exemple. Il en va de même pour la musique. Pour les arts du visible, pour la peinture, pour la photographie, la sculpture et l'architecture, il n'en va pas de même. Comme pour la poésie, leur modèle ne relève pas intégralement de leur domaine. Néanmoins, il existe au moins un exemple de système formel du visible déployé dans le langage. 


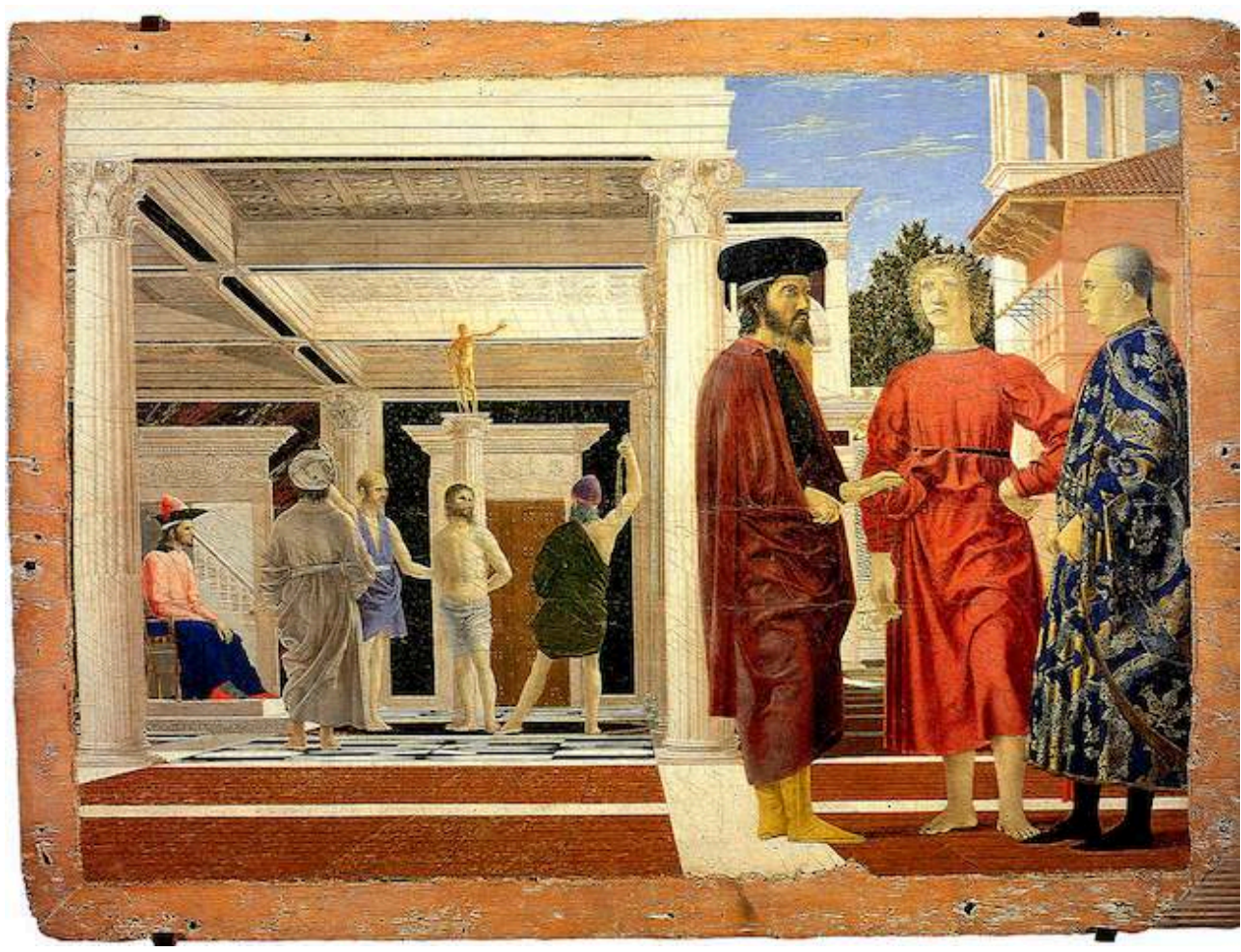

Piero della Francesca, La Flagellation du Christ, 1444-78, tempera sur peuplier, 58,4 x 81,5 cm, Urbino, Galleria Nazionale delle Marche.

Alberti rédige le De Pictura en 1435 en faisant reposer son traité en partie sur un partage entre la construction perspective du tableau et son interprétation, sa sémantique. On n'entrera pas ici dans les détails et on se contentera de souligner les moyens de l'articulation entre syntaxe et sémantique picturales. La peinture est abordée comme une science, comme une mathématique mais avec « une plus grasse Minerve ». Quel est l'enjeu du parallèle entre les mathématiques et la peinture ? Pour le problème qui nous concerne, on dira que ce parallèle permet de penser la relation entre une syntaxe et une sémantique. Cette dernière, dans le langage d'Alberti est l'istoria, ce qui est représenté par la peinture, ce dont elle parle ou, aussi bien, ce qu'elle interprète. Il ne s'agit pas d'une narration en peinture mais plutôt de la transformation en peinture, en structure du visible, d'un donné qui peut être narratif. Cette transformation suppose un lien dynamique entre les moyens de la peinture, sa syntaxe, et ce qu'elle représente. Alberti élabore donc un système formel dans lequel il y a une correspondance quasiment terme à terme entre sémantique et syntaxe. Dans son texte il nous indique comment l'on passe du plan du modèle au plan de la théorie : il y a d'abord les corps, qui composent l'istoria, puis les membres et enfin les surfaces. On est ainsi passé du plan sémantique au plan syntaxique ${ }^{16}$. Pour comprendre comment cela est possible, il faut se reporter au début du traité, quand Alberti nous explique que le nom des figures, c'est-àdire l'interprétation des corps, nous est connu par le contour, autrement dit par la syntaxe géométrique. Le début du traité se donne ainsi comme une suite de définitions géométriques, d'axiomes, qui permettent à la construction perspective de s'effectuer à la lettre ${ }^{17}$. On voit bien, par ailleurs, par les termes qu'emploie Alberti, que son souci est d'assurer la correspondance entre le domaine de la syntaxe et celui de la sémantique, le domaine des surfaces picturales et le domaine des noms. Grâce à la perspective, le 
visible se structure et l'istoria nous regarde. On peut penser au fameux exemple de $L a$ Flagellation $d u$ Christ de Piero della Francesca (fig.5): la perspective articule deux scènes, l'une qui montre l'épisode de la Flagellation du Christ et l'autre une conversation entre trois personnages contemporains du moment du tableau. Ce qui peut étonner au premier abord est l'indifférence des trois personnages du premier plan à droite, alors que dans le fond, Jésus est fouetté sous l'œil attentif de Pilate. Cet étonnement est d'autant plus fort que toute la construction perspective est élaborée de manière à créer une unité spatiale entre les deux scènes. Le mystère du tableau consiste finalement dans cette double articulation de deux espaces et de deux temps, double articulation structurée par la perspective centrale du tableau. Le modèle est donc structuré par le visible manifeste qui crée un partage entre le même espace, l'unité du tableau, et deux espaces différents, deux scènes. Si l'istoria (la structure du visible) nous regarde, elle donne figure au silence et à l'isolement, par la séparation de deux mondes, de deux temps. Nous sommes à la fois témoin de la Flagellation du Christ et de l'indifférence humaine à sa signification. L'événement de la souffrance de Jésus et de son sacrifice aura été sans incidence sur le devenir de l'humanité, il aura été vain. Ainsi le tableau de Piero n'est pas une illustration en peinture d'un récit biblique. Ce n'est pas non plus l'articulation de deux récits, mais par la dialectique de la syntaxe et de la sémantique, l'élaboration d'un rapport nouveau, complexe, de l'istoria à la présence du regard élaboré géométriquement et qui le divise ${ }^{18}$.

Ces deux exemples tendraient à montrer qu'il est possible de penser un art à l'aide des concepts des systèmes formels. Certes, Alberti n'a pas écrit son traité dans les termes des mathématiques modernes mais il rendait compte que la peinture opère à l'intersection de la syntaxe et de la sémantique. Quant à François Nicolas, nous lui devons d'avoir théorisé l'écoute musicale de manière particulièrement convaincante. Il est temps maintenant d'aborder le système formel et d'en déployer la logique telle qu'elle serait inscrite dans les photographies des modèles mathématiques. Nous aborderons d'abord la question du modèle photographique. Puis nous nous intéresserons à la syntaxe pour terminer par la généralisation que l'on peut proposer. 
Figure 6

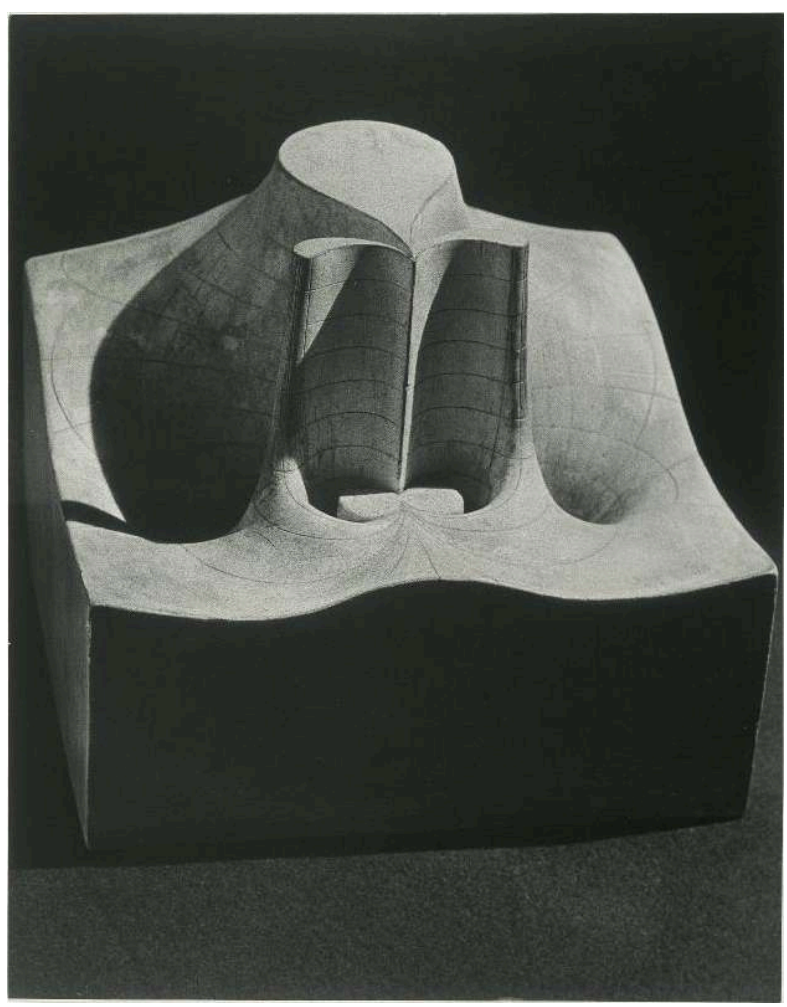

Man Ray, Objet mathématique (Expression modulaire d'une forme elliptique), 1934-36, épreuve gélatino-argentique, 30 × 24 cm, Paris, MNAM-Centre Pompidou.

https://www.centrepompidou.fr/fr/ressources/oeuvre/cgjbM7L

(c) Man Ray Trust / Adagp, Paris

Il faut commencer par cette idée que la photographie ne connaît pas les mathématiques et donc certainement pas les modèles mathématiques. Son donné est le monde des choses et leur apparence, leur aspect, notamment la chair du clair-obscur et le brillant, le poli des surfaces, l'éclat des lumières. Qu'est-ce qu'un modèle pour la photographie? Et comment en proposer une axiomatique ? On a vu que le modèle dans la formalisation mathématique était modèle mathématique, dans la musique, modèle musical. Dans la photographie il doit aussi, au moins en partie, relever de la photographie. Le deuxième élément est que le modèle est une structure, qu'il a même la structure du même et de l'autre, de l'unité et de la différence, du continu et de son prélèvement. On peut donc proposer que la photographie se constitue comme modèle de la figure qui se détache sur un fond. Cet aspect a été maintes fois souligné : les photographies de Man Ray figurent des masques, ont un caractère anthropomorphique comme la figure 6 . On peut voir ce modèle mathématique à la fois comme le haut d'un buste avec la base du cou dont on aurait tranché la tête ou un paysage vallonné, voire une architecture. Ce qui semble important est le léger basculement de la figure vers nous, grâce à la vue plongeante. Les ombres accusent les courbes, le mouvement ondulant de la surface. Enfin l'ensemble se structure comme un jeu de saillies et de creux. La figure est donc close sur elle-même. C'est une surface jaillissante et repliée, contenant ses propres principes d'orientation spatiale, autonome par rapport au fond qui, lui, disparait dans l'ombre. Rosalind Krauss, dans son étude «La Photographie au service du Surréalisme ", a insisté sur la fonction du cadre. Avec la photographie, selon elle, on constate «que la réalité se métamorphose par le moyen du cadre et que le cadre est 
perçu comme figuratif $»^{19}$. Le cadre serait pour Man Ray le moyen d'opérer sur le réel, de le transformer en modèle photographique. Mais « cadre » a dans ses photographies un sens élargi, puisqu'il n'est pas seulement l'affaire de la prise de vue. Le cadre se manifeste par la sensation que nous avons que la photographie opère par prélèvement sur le continu du visible, qu'avec elle le monde des surfaces et des étendues se replie sur lui-même. Par la force du cadre, par ailleurs, comme l'a bien analysée Rosalind Krauss, la photographie d'une configuration abstraite et informe crée une figure par ressemblance ou par dramatisation. C'est là l'un des effets structurant du cadre. On aboutit alors à la proposition que le modèle de la photographie est figuratif, comme l'est le modèle de la peinture à perspective centrale. Toutefois, alors que l'équivalence entre le point de vue et le point de fuite structure l'œuvre picturale et sa figuration, celle de la photographie provient du cadrage, de l'idée d'une découpe et d'un champ.

Figure 7

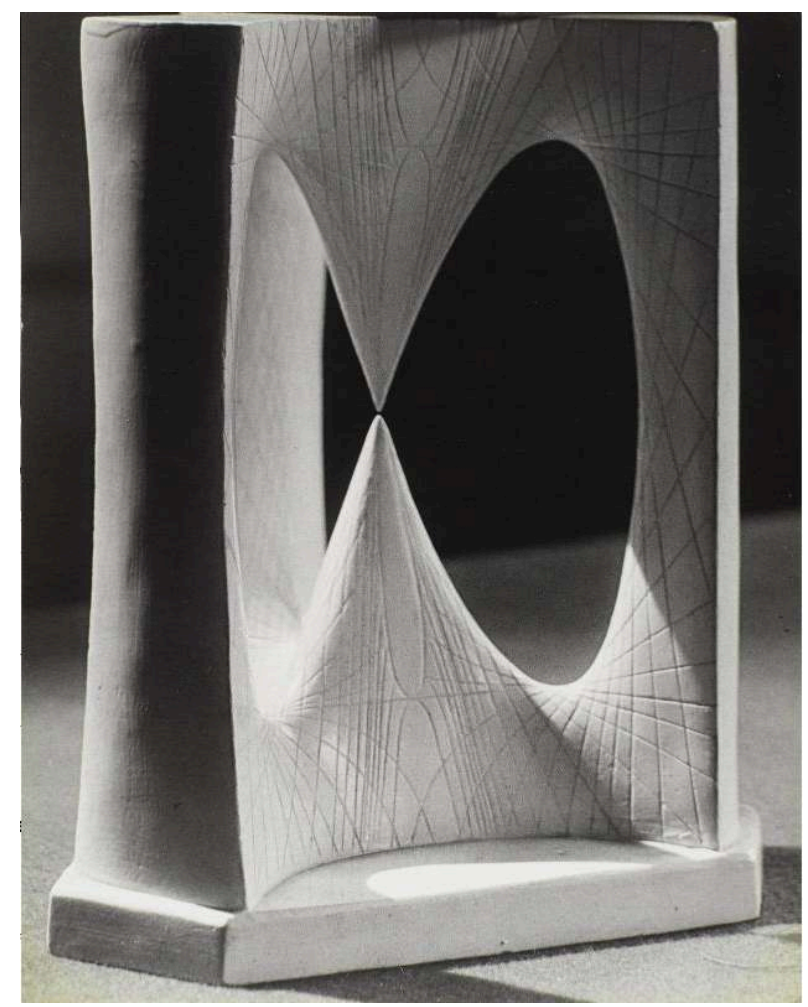

Man Ray, Objet mathématique (surface du type de celle de Cassini), 1934-36, épreuve gélatinoargentique, $30 \times 24 \mathrm{~cm}$, Paris, MNAM-Centre Pompidou.

https://www.centrepompidou.fr/fr/ressources/oeuvre/cgj4LpL

(C) Man Ray Trust / Adagp, Paris

Notre second problème porte sur la syntaxe photographique. Barthes a écrit que la photographie était un message sans code ${ }^{20}$. Nous aurions donc un modèle sans théorie, une sémantique sans syntaxe. C'est là impossible. La proposition de Barthes renvoie à la nature analogique de la photographie. Dans le système sémiotique qui est le sien, il est légitime d'écrire que la photographie dénote de manière quasiment naturelle, qu'elle enregistre le donné sans le coder. Du moins prétend-elle le faire. Néanmoins, on sait qu'une photographie est aussi l'enregistrement d'une camera obscura. Par conséquent, elle obéit aux codifications de la perspective. Rosalind Krauss, dans l'étude que nous avons mentionnée, propose une autre solution qui ferait la spécificité de la 
photographie surréaliste. «Comme les photographies sont une sorte de dépôt du réel, écrit-elle, les manipulations auxquelles les soumettent les surréalistes ont pour objet d'enregistrer les espacements et redoublements de ce même réel, dont telle photographie n'est que la trace fidèle. De cette façon, la technique photographique s'utilise pour créer un paradoxe: celui de la réalité constituée en signe $^{21}$. " La perspective demeure la même que celle de Barthes: il s'agit toujours de penser la photographie comme une sémiologie. L'idée de Krauss est que le réel offrirait à la photographie sa syntaxe, ses signifiants. Finalement, ce qu'elle nomme signe nous l'avons appelé figure et nous pensons que cela relève du modèle photographique. Le fait que le réel est continu nous détermine à cette affirmation, et un prélèvement de réel, par l'effet du cadre, n'en constitue pas une quantité discrète, mais une partie. C'est pourtant sur ces deux points, la vérité photographique et le discret, à notre avis, que s'élabore la syntaxe de la photographie. La fonction de la syntaxe dans le système formel est de produire une écriture visible, vérifiable pas à pas, pour attester d'une vérité. Une des propriétés de la syntaxe est d'être une matérialité discrète, dénombrable. On proposera que dans les photographies de Man Ray, la fonction de vérité et la propriété de discrétion est fournie par l'éclairage. Tout le travail de Man Ray à l'Institut Henri Poincaré s'est concentré sur le fait de reconstituer les conditions d'un studio avec un éclairage artificiel. Lors du tirage, les contrastes sont intensifiés, et sur presque toutes les images un éclat de lumière est visible. Cet éclat, comme pour la photographie de la surface du type de Cassini, s'amenuise jusqu'à devenir la pointe d'une pique (voir la figure 7). Ainsi, la lumière, l'éclairage visible des photographies, viendrait attester de l'enregistrement photographique et de son apparition pas à pas. De la même manière que le modèle mathématique est montré déterminé par les courbes graphiques qui l'enserrent, le modèle photographique est construit par son éclairage qui lui donne forme. Ce dernier atteste de l'opération syntaxique de la photographie.

On peut maintenant résumer la double opération, sémantique et syntaxique, selon laquelle la photographie a lieu. Il y a d'une part l'élaboration d'un modèle, continu, qui est la figure qui s'enlève sur un fond par l'effet du cadrage et d'autre part le développement d'une syntaxe dans l'éclairage photographique.

Parvenu en ce point où la dialectique entre le modèle et la théorie a été saisie, il nous faut généraliser notre propos. Il apparaît que la photographie relève d'une sémantique figurative que sert une syntaxe de la lumière. Cela signifie que la figure est aussi créée par la lumière. Il n'est pas suffisant de dire que la figure est produite au niveau phénoménal par la lumière, qu'elle est révélée, mais bien qu'elle est créée par une cause abstraite. Il se peut que Breton ait senti cela. Il semble en tous cas que le problème de l'abstraction de ces figures ait été au cœur des échanges entre Breton et Man Ray. L'échange ne portait pas sur le fait que ce soient des objets mathématiques et abstraits mais bien que les photographies se montrent elles-mêmes comme abstraites, qu'en quelque sorte, se mesurant à l'abstraction mathématique, elles révèlent la part abstraite du phénomène lumineux. Dans une lettre que Man Ray écrit à Breton, et qui servira de préface à une exposition de ses peintures intitulées Équations shakespeariennes, Man Ray revient sur la prétendue abstraction de ses photographies. Selon lui, telle n'a jamais été sa volonté. Mais il ajoute qu'il a toujours pris un plaisir diabolique à l'embarras dans lequel elles jetaient les tenants de l'art abstrait. On peut donc dire que le démenti est aussi l'aveu de leur dimension abstraite. 
Figure 8

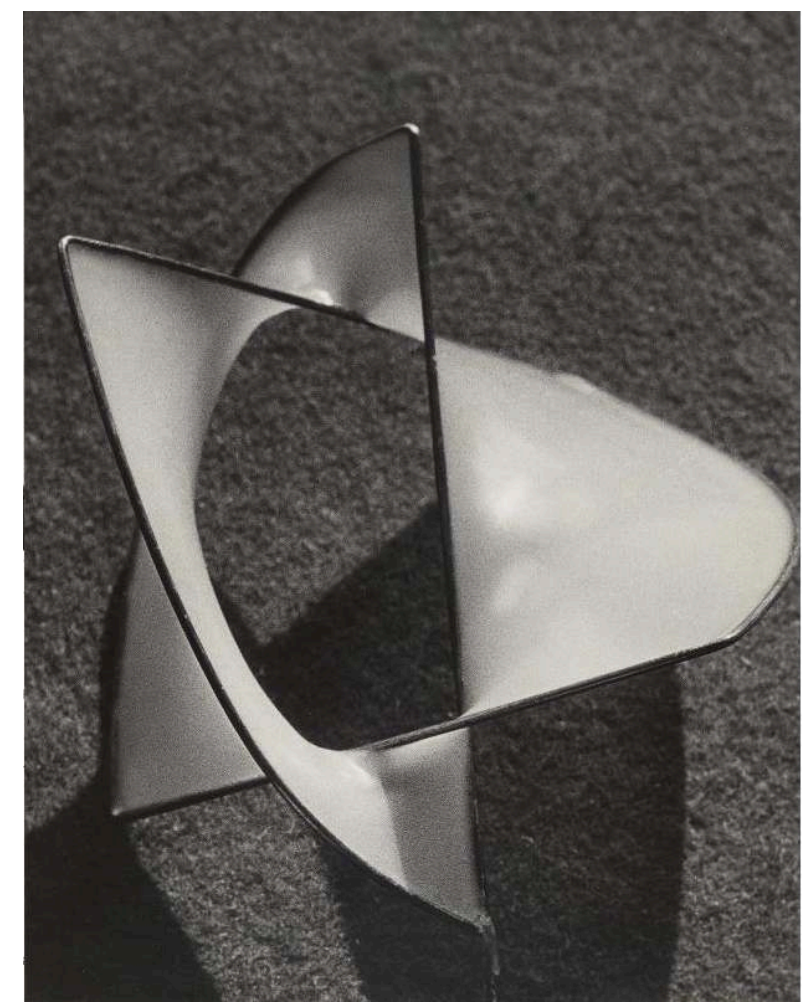

Man Ray, Objet mathématique, 1934-36, épreuve gélatino-argentique, 30 × 24 cm, Paris, MNAM-Centre Pompidou.

https://www.centrepompidou.fr/fr/ressources/oeuvre/c9nX5jk

(c) Man Ray Trust / Adagp, Paris

Reprenons les éléments. Breton oppose abstraction et concrétion dans son essai «Crise de l'objet ». Toutefois les Objets mathématiques ne se posent pas en ces termes. Ils montrent des objets qui appartiennent à une réalité empirique, des objets du monde naturel, comme peut l'être le modèle mathématique de la figure 8 aussi aérien et léger qu'une libellule, ou charnels comme les deux corps ajustés qui s'attirent et s'écartent l'un de l'autre de la figure 1. Mais en même temps, tout nous montre que cette réalité est construite : les objets, dont on perçoit l'ossature ou l'armature technique ${ }^{22}$, et la photographie qui impose toujours sa manipulation. Les photographies de Man Ray se situent donc à l'articulation des deux mondes du donné naturel et du construit, du continu et du discontinu (que l'on regarde à nouveau la figure 6 qui alterne courbes des surfaces et courbes tracées qui semblent continues, et le discontinu des trous et des arêtes dramatisé par le cou coupé). Elles en font même un principe structurant de la photographie moderne. Ainsi, l'interdépendance de la dialectique entre la théorie et le modèle nous dit que chaque art est spécifique, qu'il provient du nouage particulier, historiquement vérifiable entre une syntaxe et une sémantique. L'on voit aussi que la dialectique formelle exhibée dans les Objets mathématiques traverse toute une partie de l'art moderne qui ne s'est pas contenté de reproduire le réel ou de le documenter, depuis la fin du XIX ${ }^{e}$ siècle jusqu'aux années quarante.

Enfin, une dernière remarque qui veut formuler une espérance dans le travail sur les formes. Man Ray s'attache à photographier les interprétations sémantiques, les modèles, d'une syntaxe mathématique. Il photographie donc le mouvement qui mène 
de l'abstraction au concret. Mais dans le même temps, dans le même mouvement, ses photographies attestent et formalisent la direction inverse, celle qui, prenant appui sur le concret du monde, en élabore une syntaxe abstraite. Ce développement de la syntaxe propre à la photographie a su créer des objets nouveaux, des formes nouvelles, a su étendre le possible. C'est exactement ce que Breton souhaitait, mais selon une autre logique que celle dont il s'était fait le héraut. Selon lui, le possible à conquérir était celui de l'imaginaire et de ses secrets. À la même époque, Man Ray montrait dans ses photographies que le possible consistait à développer de nouvelles formes, de nouvelles manières de composer des œuvres. Un tel possible, formel, est toujours ouvert.

\section{NOTES}

1. Les Cahiers d'art, t. 11, 1-2, 1936, p. 4-10. Pour une étude et une présentation des photographies de Man Ray, on se reportera à Emmanuelle DE L'ECOTTAIS, Alain SAYAG (dir.), Man Ray, la photographie à l'envers, catalogue de l'exposition. Paris (Centre Pompidou), Paris, Éditions du Centre Georges Pompidou - Seuil, 1998; Isabelle FORTUNÉ, «Man Ray et les objets mathématiques ", Études photographiques, t. 6, mai 1999 ; ainsi qu'au catalogue d'exposition réalisé sous la direction de W. GROSSMAN et E. SEBLINE, Man Ray. Human Equations: A Journey from Mathematics to Shakespeare, catalogue de l'exposition. Washington, DC (The Phillips Collection), Ostfildern, Hatje Cantz Verlag, 2015.

2. André BRETON, "Crise de l'objet", Cahiers d'Art, t.1-2, mai 1936, p. 21-26, repris dans Le Surréalisme et la peinture, Paris, Gallimard, 1965, p. 353-360 (citation p. 355). Il est à noter que dans cette ré-édition de son essai, Breton a supprimé l'allusion aux photographies de Man Ray, ce qui renforce bien le rôle secondaire qu'elles jouent dans sa réflexion.

3. Ibid., p. 356.

4. Voir respectivement l'ouvrage collectif de l'Institut Henri Poincaré, Objets mathématiques, Paris, CNRS Éditions, 2017 ; et Emmanuel GUIGON (dir.), Objets mathématiques, catalogue de l'exposition. Besançon (Musée du Temps), Milan, Silvana Editoriale - Besançon, Université de Franche-Comté, 2014.

5. Voir François APÉRY, « La collection », in Objets mathématiques (2017), op. cit., p. 12.

6. Elle cite ce passage de Henry Vuibert, Les anaglyphes géométriques, Paris, Vuibert, 1912, p. 13 : «Pour aider les élèves à voir dans l'espace, on a matérialisé les principales figures de la géométrie et de la géométrie descriptive. L'emploi de figures en relief apporterait un secours précieux à l'enseignement, surtout si l'on faisait construire ces figures par les élèves. »

7. Alain BADIOU, Le Concept de modèle, Paris, Fayard, 2007 [1969].

8. En plus du livre de Badiou déjà cité, une présentation du système formel est donnée dans Roger MARTIN, Logique contemporaine et formalisation, Paris, P.U.F, 1964.

9. Le schéma a été élaboré par François Nicolas et a été expliqué lors de la $6^{\mathrm{e}}$ leçon de mathématiques donnée lors de la séance Qui-vive du 16 mai 2016. La leçon complète est consultable sur le site suivant : http://www.egalite68.fr/H68/Bulletin-6.pdf, consulté le 23 février 2020.

10. R. MARTIN, Logique contemporaine, op. cit., p. 27.

11. A. BADIOU, Le Concept de modèle, op. cit., p. 125. 
12. François NICOLAS, Le monde-Musique, 2 t., Paris, Aedam Musicae, 2014. Voir particulièrement le tome 2.

13. A. BADIOU, Le Concept de modèle, op. cit., p. 133.

14. Sur la dialectique et le dynamisme du système formel, voir A. BADIOU, ibid., notamment p. 107 : « Une structure est modèle d'une théorie formelle si tous les axiomes de cette théorie sont valides pour cette structure. " Le modèle n'est pas un donné inerte. Du fait de sa relation avec une théorie, le modèle est une structure, c'est-à-dire qu'y opère la dialectique du même et de l'autre qui permet d'inscrire une correspondance entre les relations internes à la syntaxe et celles internes au modèle.

15. On ne peut que renvoyer aux remarquables deux premiers volumes de F. NICOLAS, Le mondeMusique, op. cit., notamment aux chapitres 2 et $6 \mathrm{du} 1^{\mathrm{er}}$ volume, et les chapitres 2 à $5 \mathrm{du} 2^{\mathrm{e}}$ volume. Il est dommage qu'il n'existe pas d'édition plus brève, plus ramassée sur ce sujet, et plus accessible tant cette étude de la formalisation musicale peut être une source de suggestions dans de nombreux domaines. Elle nous a particulièrement inspiré ici.

16. Voir Léon Battista ALBERTI, La Peinture, trad. du latin et de l'italien par Thomas Golsenne et Bertrand Prévost, Paris, Seuil, 2004 [De Pictura, Florence, 1435 ; Della Pittura, Florence, 1436]. Le passage en italien est le suivant : «Grandissima opera del pittore sarà l'istoria : parte della istoria sono $i$ corpi : parte de' corpi sono i membri : parte de' membri sono le superficie. » Ibid., p. 240.

17. «Adunque l'orlo e dorso danno suoi nomi alle superfie. » Ibid., p. 214.

18. Le visible mis en œuvre par la perspective n'est pas le visible naturel, tel qu'il se donne dans la vision. En témoigne le fait que la perspective aura réduit la vision à un seul œil, alors que nous en avons deux pour voir. Sur ce point, il faut lire l'ouvrage d'Hubert DAMISCH, L'Origine de la perspective, Paris, Flammarion, 1987.

19. Rosalind KRAUSS, « La Photographie au service du Surréalisme », dans Rosalind KRAUSS, Jane LIVINGSTON, Dawn ADES, Explosante-fixe. Photographie et Surréalisme, trad. de l'anglais par Dominique Le Bourg et Dominique Saran, catalogue de l'exposition. Paris (Musée national d'art moderne), Paris, Hazan, 2002 [éd. orig. L'amour fou: Photography and Surrealism, Washington, DC, Corcoran Gallery of Art, New York, Abbeville Press, 1985], p. 19.

20. Roland BARTHES, «Rhétorique de l'image», Communications, t.4, 1964, p.40-51. Voici le passage : «Ce caractère utopique de la dénotation est considérablement renforcé par le paradoxe qu'on a déjà énoncé et qui fait que la photographie (dans son sens littéral), en raison de sa nature absolument analogique, semble bien constituer un message sans code.» Repris dans Roland BARTHES, Euvres complètes (1962-1967), t. 2, Paris, Seuil, p. 581.

21. R. KRAUSS, « La Photographie au service du Surréalisme », op. cit., p. 31.

22. À propos des objets qu'il a photographiés, Man Ray écrit : «I took new courage in my resolve to seek inspiration as much as possible from made-man objects. » Man Ray, "A Note on the Shakspearean Equations ", 1948, repris dans Man Ray, Writings on Art, édités par Jennifer Mundy, The Getty Research Institute, 2015, p. 340-345, citation p. 340.

\section{RÉSUMÉS}

Les photographies des objets mathématiques faites par Man Ray sont mystérieuses. Leur mystère tient autant aux objets photographiés qu'aux photographies elles-mêmes. On propose alors de saisir ce mystère comme une pensée de la photographie sur elle-même. Il apparaît donc possible 
d'expliquer la logique photographique à la lumière de la logique formelle des mathématiques, notamment à celle de la théorie des modèles, telle qu'elle se montre dans les objets mathématiques.

Man Ray's photographs of mathematical objects are mysterious. Their mystery lies as much in the objects photographed as in the photographs themselves. We then propose to grasp this mystery as a thought of the photograph on itself. It seems possible to explain the photographic logic in the light of the formal logic of mathematics, especially the model theory, as it is shown in the mathematical objects.

\section{INDEX}

Mots-clés : Man Ray - Objets mathématiques - Photographie - Modèle - Formalisation

Keywords : Man Ray - Mathematical objects - Photography - Modell - Modelling

\section{AUTEUR}

\section{ÉRIC BRUNIER}

Professeur de lettres modernes, doctorant au Centre de Recherches sur les Arts et le Langage (CRAL - École des Hautes Études en Sciences Sociales). Il mène un travail de recherche sur les relations, au sein du tableau, entre le trait, la couleur et la surface (travail sur Lucio Fontana, le cubisme). Par ailleurs il s'intéresse au discours du tableau dans la peinture classique (Poussin). Il a organisé plusieurs expositions d'art contemporain avec le Casino Luxembourg - Forum d'Art Contemporain. 